\title{
Epithelial-Mesenchymal Transition (EMT) and metastasis: yes, no, maybe?
}

\author{
Maren Diepenbruck and Gerhard Christofori
}

Department of Biomedicine, University of Basel, 4058 Basel

$\begin{array}{ll}\text { Corresponding author: } & \text { Gerhard Christofori } \\ & \text { Department of Biomedicine } \\ & \text { University of Basel } \\ & \text { Mattenstrasse } 28 \\ & 4058 \text { Basel } \\ & \text { Switzerland } \\ & \text { Tel. }+41612673564 \\ & \text { Fax. }+41612673566 \\ & \text { E-mail: Gerhard.christofori@unibas.ch }\end{array}$

Running title: EMT and Cancer

Keywords: cancer, EMT, metastasis, therapy resistance

Word Count: 2749

\section{Abstract}

An epithelial to mesenchymal transition (EMT) is a process of cell remodeling critical during embryonic development and organogenesis. During an EMT, epithelial cells lose their polarized organization and acquire migratory and invasive capabilities. While a plethora of experimental results have indicated that manipulating an EMT also affects cancer metastasis, its reverse process, a mesenchymal to epithelial transition (MET), seems to support metastatic outgrowth in distant organs. Moreover, recent reports investigating cancer cells circulating in the blood stream or employing genetic lineage-tracing have questioned a critical role of an EMT in metastasis formation. Hence, we need to better understand the molecular networks underlying the cell plasticity conferred by an EMT or a MET and its functional contribution to malignant tumor progression. 


\section{Introduction}

The malignant form of cancer and the cause for more than $90 \%$ of cancer-associated deaths is characterized by a cancer cell's ability to invade into the surrounding tissue, to disseminate throughout the body and to establish secondary tumors (metastases) at distant organs [1]. The "invasion-metastasis cascade" (Figure 1) describes the sequential processes leading to the formation of metastasis in distant organs. It starts with a loss of adhesion of tumor cells and their migration out of the primary tumor and invasion into the surrounding tissue. Subsequently, cells intravasate into the blood or lymphatic circulation. When disseminating throughout the body, cancer cells have to survive the harsh conditions in the bloodstream and cope with shearing forces, anoikis and immune surveillance. At a secondary site, cells adhere to the target organ endothelium, extravasate and migrate into the organ parenchyma [2]. Upon reaching the "metastatic niche", they either enter a longlasting dormant cell state as individual disseminated tumor cells (DTCs) [3] or as multicellular micrometastases [4] which eventually start to proliferate to form macroscopic metastases [5]. It has been estimated that only $0.01 \%$ of tumor cells entering the systemic circulation are able to form secondary tumors [6].

The multi-stage process of an EMT comprises the gradual remodeling of epithelial cell architecture and functional capabilities. Cells lose their epithelial cell-cell junctions and apical-basal cell polarity and convert to a low proliferation state with spindle-like cell shape and with increased cell migration, invasion and survival. The aberrant activation of an EMT promotes tumor cell invasion and dissemination, while its reverse process, a mesenchymal to epithelial transition (MET), is believed to support metastatic outgrowth once cancer cells have arrived in distant organs [7-9]. Apparently, the cell plasticity mediated by the EMT/MET processes is an indispensable feature during physiological and pathological processes, such as embryogenesis, tissue homeostasis, tissue fibrosis and cancer.

\section{Molecular networks regulating an EMT}

Various extracellular stimuli can activate signaling cascades that mediate EMT progression during development, tissue regeneration and malignant tumor progression. A variety of classical growth factors can induce EMT in a contextdependent manner. These factors are not only secreted by tumor cells, but also by cells of the tumor stroma. Other signaling pathways, such as Wnt, Notch, Hedgehog, JAK-STAT, AP-1, NF-KB and Hippo signaling also induce or modulate the EMT 
process [7-9]. Metabolic and mechanical stresses, tissue hypoxia or matrix stiffness can also act as powerful inducers of EMT and tumor cell invasion [10].

The majority of these EMT-inducing pathways converge on the level of EMTassociated transcription factors (TFs) [11]. The TF families Snail (zinc finger proteins Snail and Slug), Zeb (zinc finger and homeodomain proteins Zeb1 and Zeb2) and Twist (basic helix-loop-helix proteins E12, E47, Twist1, Twist2 and Id) play a central role during a developmental and oncogenic EMT and are considered master EMT TFs [12]. They are potent inducers of the epithelial cell de-differentiation process by acting as transcriptional repressors of epithelial genes, including E-cadherin, and activators of mesenchymal genes, including N-cadherin [10,12]. Other TFs have been shown to control or contribute to EMT/MET cell plasticity, including but not limited to Prrx1 [13], Sox4 and Sox9 [14,15], Klf4 [16], FoxC2 [17], p53 [18], members of the AP-1 (Jun/Fos) family [19], and Tead2 [20], and the list keeps growing.

Of the many miRNAs changing in their expression during an EMT, members of the miR-200 family are associated with epithelial cell morphology and their expression is decreased upon the induction of an EMT [21-24]. Ectopic expression of individual family members blocks an EMT or induces a MET in mesenchymal cells by posttranscriptional repression of the EMT TFs Zeb1 and Zeb2 [23,25]. Conversely, Zeb1/2 directly bind to miR-200 promoters and repress their expression. This reciprocal regulation of Zeb1/2 and miR-200 family members not only determines cell morphology but also controls cell migration and invasion [26,27]. A number of such double-negative feedback loops between miRNAs and key EMT TFs function as molecular switches and are important mechanisms underpinning the fine-tuning and reversibility of an EMT and, thus, epithelial/mesenchymal cell plasticity [27-29].

In conclusion, numerous TFs and miRNAs, epigenetic modifications, long non-coding RNAs, circular RNAs and alternative splicing factors appear to control the process of an EMT, yet the epistatic hierarchy of these regulatory networks still needs to be established.

\section{What is the evidence for an EMT in malignant cancer progression?}

Over the past decade and with the development of novel technologies, numerous reports on the cellular processes and molecular players and pathways critical in metastasis have reported seemingly conflicting views. The ongoing discussions culminate in the question whether an EMT is required for metastasis to occur. As a first step to adequately integrate these conflicting data we need to dissect the stages of the metastatic cascade. For example, it is important to distinguish various 
experimental settings frequently used in metastasis research. The formation of metastases from established primary tumor obviously requires all stages of the metastatic cascade, notably primary tumor cell invasion and intravasation as single cell or as cell collective, their dissemination in the blood stream, extravasation and, finally, metastatic outgrowth. In contrast, the direct injection of cancer cells into the blood circulation only needs outgrowth in the target organ, while the need for an active extravasation is often ignored. Whether and when an EMT or a MET actually occurs has only recently begun to be analyzed in a temporal and spatial manner, for example by intravital imaging and/or by genetic lineage tracing experiments. Here, we summarize some of the key points that could help to integrate the conflicting data into a working model.

A plethora of in vitro and in vivo studies have revealed that an EMT promotes cancer cell migration and invasion into the stroma [7,30]. Cells at the invasive tumor front often display a de-differentiated morphology that is accompanied by a loss of epithelial markers and cell-cell junctions and a gain of the expression of mesenchymal markers [31,32]. Experimentally, the induction of EMT in cancer cells, for example by TGF $\beta$ signaling, by the forced expression of EMT TFs or by interfering with E-cadherin function, has lead to an increase in metastasis. Conversely, inhibition of an EMT, for example by siRNA-mediated knockdown or genetic knockout of EMT-inducing genes, or the induction of a MET, for example by the expression of an epithelial differentiation inducer, has resulted in reduced metastasis. In conclusion, numerous reports convey the notion that whenever the function of a gene implicated in an EMT is modulated, metastasis formation is affected as well $[30,33,34]$. Furthermore, circulating tumor cells (CTCs) isolated from the bloodstream of patients with metastatic breast cancers exhibit more mesenchymal cell marker expression as compared to primary tumor cells, and this mesenchymal marker expression correlates with poor clinical outcome [35,36]. These findings are supported by in vivo lineage tracing of tumor cells in a Kras/p53driven mouse model of pancreatic cancer in which invading and circulating pancreatic tumor cells exhibit a rather mesenchymal cell phenotype [37]. Moreover, tumor differentiation status appears to correlate with clinical outcome in patients, where less differentiated tumors indicate a poorer survival prognosis, for example in breast cancer patients [38]. Finally, an EMT has been correlated with cancer stem cell traits and with increased tumorigenicity and drug resistance, all hallmarks of metastatic cells $[39,40]$. As a consequence, for the past years the research field has been satisfied with the notion that EMT is a critical process in the metastatic process. 
In a first escalation of the discussion, recent reports indicate that an EMT may only be critical for the initial steps of the metastatic cascade up to organ extravasation, but not for the metastatic outgrowth at the distant site. These data demonstrate the importance of a reversible EMT/MET dynamics for metastatic outgrowth. For example, activation of Twist1, a classical EMT inducer, in the primary tumor induces tumor cell intravasation and blood stream dissemination in a mouse model of squamous cell carcinoma. Yet, it is Twist1 inactivation and thereby induction of a MET at the metastatic site that is crucial for metastatic outgrowth [41]. Similar observations have been reported for the other EMT/MET inducing factors Prrx1 [13], Id1 [42] and Snail [43]. Furthermore, an MET at the secondary sites would explain why metastatic lesions often display a differentiated phenotype comparable to the corresponding primary tumors, and not a highly mesenchymal-like and invasive phenotype that is only observed in metastases of certain cancer types [44].

To integrate the novel observations, two potential types of metastasis formation have been suggested [45]. The first model is referred to as "plasticity type I" metastasis model, in which tumor cells are susceptible to signals from the microenvironment and where dynamic EMT/MET processes drive metastasis formation. The second model, the "genetic type II" metastasis model, proposes that tumor cells undergo a permanent, irreversible EMT, exhibit stem-like properties and lose their phenotypic plasticity upon genetic alterations. Such genetic alterations force cancer cell metastatic colonization and outgrowth without the need for an MET [45]. The first model, increased EMT/MET plasticity - or in other words, a partial EMT or a hybrid epithelial/mesenchymal phenotype [46] - is characterized by the coexistence of epithelial and mesenchymal markers in a cancer cell and is supported by the recent use of novel analytical technologies. For example, intravital imaging technologies have demonstrated induction of an EMT in migrating and intravasating tumor cells by growth factors released by inflammatory cells, such as macrophages $[47,48]$. Moreover, the analysis of CTCs in the blood stream of patients and of experimental mouse models has revealed the concomitant expression of mesenchymal and epithelial genes in individual cells but also in clusters of CTCs [35] and also in DTCs in the bone marrow of patients [49]. Initially, these investigations have been confounded by the use of epithelial markers to identify CTCs and DTCs, such as EpCAM or cell type-specific cytokeratins.

More recently, label-free isolation of CTCs in microfluidic devices and/or hand-picking by micromanipulators has revealed very rare CTCs in patients with various cancer types. Notably, while CTCs are found mainly as single cells, they are also detected in clusters. These CTC clusters are more tumorigenic and metastatic 
as compared to single CTCs [50-52]. There is the argument that clusters of 20 or more CTC should not be able to pass through capillaries and should be rapidly trapped in microcirculation. Yet, a recent report shows that CTC clusters can pass through microfluidic devices and through the capillaries of Zebrafish by a rapid and reversible reorganization into single-file chains [53]. Notably, single CTCs exhibit a mixed expression of epithelial and mesenchymal markers suggesting a hybrid E/M phenotype. Also the CTC clusters seem to express epithelial and mesenchymal markers, yet they have not been analyzed at a single cell level to distinguish whether they are composed of epithelial and mesenchymal cells or whether individual cells within the CTC cluster simultaneously express epithelial and mesenchymal markers. It is important to note that desmosomal junction proteins, such as plakoglobin, seem required for an efficient metastatic outgrowth of CTC clusters. The mechanistic implications of this observation warrant further investigation.

Cells concomitantly expressing epithelial and mesenchymal genes seem predominant in many cancer types, including aggressive melanoma and breast cancers [54,55]. Thus, a hybrid E/M phenotype of cancer cells may mean a worse prognosis for patients as compared to an exclusive mesenchymal phenotype. However, the E/M phenotype has not been specifically analyzed, whether it is metastable or whether it confers to the cell a specific plasticity to adapt to other environments and therapy [8]. Using elegant fate mapping experiments by intravital microscopy, this epithelial/mesenchymal plasticity has been shown to be critical for metastasis formation: mesenchymal EMT cells arriving at the secondary site rapidly convert to an epithelial state to form macrometastasis [56]. These results indicate that EMT plasticity is required for early migration, but upon arrival at the secondary site mesenchymal-like cancer cells revert to epithelial cells and efficiently form metastases.

The detection of rare clusters of CTCs in the blood stream of patents begs the question whether these cells have escaped from the primary tumors as cluster or whether they have formed the cluster later on by single CTCs. Elegant combinations of lineage tracing and tumor transplantation experiments have revealed that these clusters do not form in the blood stream and are originating from polyclonal primary tumors. Most importantly, they form polyclonal metastasis at distant sites [50,57]. These results raise the possibility that tumor cells may detach from the primary tumor by collective migration characterized by the simultaneous migration of cells that are still attached to each other by cell-cell adhesions. Collective cell migration is for example a hallmark of squamous cell carcinomas of various tissues, including head and neck, cervix and lung. Here, the leading cells of finger-like protrusions invading 
into the tumor stroma express the cell surface protein podoplanin which is able to induce filopodia formation and cell migration [58]. Similarly, expression of podocalyxin has been reported to induce collective migration in breast cancer cell lines [59]. Notably, these cells are still connected to their neighbors by E-cadherinmediated cell junctions. Whether an EMT is required for this process - at least in the leading cells - is still debated. In fact, recent reports demonstrate that the cancer cells leading collective invasion in the major types of human breast cancers express basal epithelial genes, including E-cadherin, cytokeratin 14 and p63 [60]. Notably, these cellular features are also found in CTC clusters, and depletion of CK 14 and p63 disrupts the expression of well-known metastasis genes, such as Tenascin-C, Jagged 1 and Epiregulin and prevents metastasis [61]. Other cancers may also exhibit collective migration, for example in colorectal cancer the budding of tumor cells at the invasive front correlates with poor clinical outcome [62].

The discussion about the role of EMT in cancer metastasis does not end here. Recent lineage tracing experiments have cardinally questioned the functional contribution of an EMT to the metastatic process, and rather highlighted the importance of an EMT for resistance against chemotherapy. One study has shown that EMT seems to occur predominantly in Myc-driven breast carcinogenesis and not so much in other oncogene-driven breast cancers, not only in mice but also in patients. An important conclusion of this work is that the extent of EMT does not correlate with metastasis [63]. In two more recent reports, lineage tracing and the genetic ablation of the major EMT TFs Snail or Twist in mouse models of breast cancer and pancreatic cancer, respectively, have been used to demonstrate that EMT is not relevant for metastatic outgrowth but for chemotherapy resistance $[64,65]$. The latter two reports have cast serious doubts on the functional contribution of an EMT to the metastatic process, yet they have also been met with cautious skepticism. For example, the use of gene promoters for lineage tracing that are known to be highly active in bona fide fibroblasts (Fsp1) or in late stage EMT (vimentin) may miss a potential function of a partial or early EMT in metastasis formation. Moreover, the genetic ablation of only one individual EMT TF, such as Snail or Twist, may be compensated by the other EMT-TFs [66]. Most recently, Fsp1 promoter-driven lineage tracing of breast cancer cells has been used for intravital imaging, showing that the transplanted cells can undergo an EMT that can be pharmacologically targeted, for example with an inhibitor against the HGF-receptor cMet [67]. The latter report also raises the possibility that transplantation models may behave differently from endogenously growing tumors when it comes to tumor cells undergoing an EMT. After all, it is assumed that the inductive signals for an EMT 
come from the tumor stroma which certainly differs between experimental settings and cancer types.

\section{Conclusions}

The dynamic nature of cellular EMT and MET processes are essential during embryogenesis and wound healing, since they promote organ formation and tissue regeneration. In the past years, it has been widely accepted that during tumorigenesis aberrant activation of these networks can induce oncogenic EMT, which promotes tumor cell invasion and malignant tumor progression. However, recent experimental results have raised concerns about the actual contribution of an EMT to metastasis formation. Rather an EMT may be part of a cellular status of high cell plasticity that allows cells to escape chemotherapy, hypoxia and other impediments. Hence, we need to take a step back, reconsider some of our views and resolve the current dispute by scientific approaches, i.e. adequate experimentation. For example, a fate mapping experiment in which tumor cells are specifically marked and then monitored for an EMT during malignant tumor progression in vivo has still to be performed.

\section{Acknowledgments}

We apologize to all colleagues whose important work could not be cited due to space limitations. The research in the laboratory of the authors has been supported by the SystemsX.ch RTD project Cell Plasticity, the Swiss Cancer League, and the Swiss National Science Foundation.

\section{References}

1. Gupta GP, Massague J: Cancer metastasis: building a framework. Cell 2006, 127:679-695.

2. Massague J, Obenauf AC: Metastatic colonization by circulating tumour cells. Nature 2016, 529:298-306.

3. Klein CA: Parallel progression of primary tumours and metastases. Nat Rev Cancer 2009, 9:302-312.

4. Sosa MS, Bragado P, Aguirre-Ghiso JA: Mechanisms of disseminated cancer cell dormancy: an awakening field. Nat Rev Cancer 2014, 14:611-622.

5. Chaffer CL, Weinberg RA: A perspective on cancer cell metastasis. Science 2011, 331:1559-1564.

6. Chambers AF, Groom AC, MacDonald IC: Dissemination and growth of cancer cells in metastatic sites. Nat Rev Cancer 2002, 2:563-572.

7. Thiery JP, Acloque H, Huang RY, Nieto MA: Epithelial-mesenchymal transitions in development and disease. Cell 2009, 139:871-890. 
8. Nieto MA: Epithelial plasticity: a common theme in embryonic and cancer cells. Science 2013, 342:1234850.

9. Acloque H, Adams MS, Fishwick K, Bronner-Fraser M, Nieto MA: Epithelialmesenchymal transitions: the importance of changing cell state in development and disease. J Clin Invest 2009, 119:1438-1449.

10. Lamouille S, Xu J, Derynck R: Molecular mechanisms of epithelialmesenchymal transition. Nat Rev Mol Cell Biol 2014, 15:178-196.

11. Nieto MA, Cano A: The epithelial-mesenchymal transition under control: global programs to regulate epithelial plasticity. Semin Cancer Biol 2012, 22:361-368.

12. Peinado $H$, Olmeda $D$, Cano $A$ : Snail, Zeb and bHLH factors in tumour progression: an alliance against the epithelial phenotype? Nat Rev Cancer 2007, 7:415-428.

13. Ocana OH, Corcoles R, Fabra A, Moreno-Bueno G, Acloque H, Vega S, BarralloGimeno A, Cano A, Nieto MA: Metastatic colonization requires the repression of the epithelial-mesenchymal transition inducer Prrx1. Cancer Cell 2012, 22:709-724.

14. Tiwari N, Tiwari VK, Waldmeier L, Balwierz PJ, Arnold P, Pachkov M, MeyerSchaller N, Schubeler D, van Nimwegen E, Christofori G: Sox4 is a master regulator of epithelial-mesenchymal transition by controlling Ezh2 expression and epigenetic reprogramming. Cancer Cell 2013, 23:768-783.

15. Guo W, Keckesova Z, Donaher JL, Shibue T, Tischler V, Reinhardt F, Itzkovitz S, Noske A, Zurrer-Hardi U, Bell G, et al.: Slug and Sox9 cooperatively determine the mammary stem cell state. Cell 2012, 148:1015-1028.

16. Tiwari N, Meyer-Schaller N, Arnold P, Antoniadis $H$, Pachkov M, van Nimwegen $\mathrm{E}$, Christofori G: KIf4 is a transcriptional regulator of genes critical for EMT, including Jnk1 (Mapk8). PLoS One 2013, 8:e57329.

17. Mani SA, Yang J, Brooks M, Schwaninger G, Zhou A, Miura N, Kutok JL, Hartwell K, Richardson AL, Weinberg RA: Mesenchyme Forkhead 1 (FOXC2) plays a key role in metastasis and is associated with aggressive basal-like breast cancers. Proc Natl Acad Sci U S A 2007, 104:10069-10074.

18. Chang CJ, Chao CH, Xia W, Yang JY, Xiong Y, Li CW, Yu WH, Rehman SK, Hsu $\mathrm{JL}$, Lee $\mathrm{HH}$, et al.: p53 regulates epithelial-mesenchymal transition and stem cell properties through modulating miRNAs. Nat Cell Biol 2011, 13:317-323.

19. Bakiri L, Macho-Maschler S, Custic I, Niemiec J, Guio-Carrion A, Hasenfuss SC, Eger A, Muller $M$, Beug $H$, Wagner EF: Fra-1/AP-1 induces EMT in mammary epithelial cells by modulating Zeb1/2 and TGFbeta expression. Cell Death Differ 2015, 22:336-350.

20. Diepenbruck M, Waldmeier L, Ivanek R, Berninger P, Arnold P, van Nimwegen E, Christofori G: Tead2 expression levels control the subcellular distribution of Yap and Taz, zyxin expression and epithelial-mesenchymal transition. $J$ Cell Sci 2014, 127:1523-1536.

21. Zavadil J, Narasimhan M, Blumenberg M, Schneider RJ: Transforming growth factor-beta and microRNA:mRNA regulatory networks in epithelial plasticity. Cells Tissues Organs 2007, 185:157-161.

22. Kong W, Yang H, He L, Zhao JJ, Coppola D, Dalton WS, Cheng JQ: MicroRNA155 is regulated by the transforming growth factor beta/Smad pathway and contributes to epithelial cell plasticity by targeting RhoA. Mol Cell Biol 2008, 28:6773-6784.

23. Gregory PA, Bert AG, Paterson EL, Barry SC, Tsykin A, Farshid G, Vadas MA, Khew-Goodall Y, Goodall GJ: The miR-200 family and miR-205 regulate epithelial to mesenchymal transition by targeting ZEB1 and SIP1. Nat Cell Biol 2008, 10:593-601. 
24. Diaz-Martin J, Diaz-Lopez A, Moreno-Bueno G, Castilla MA, Rosa-Rosa JM, Cano A, Palacios J: A core microRNA signature associated with inducers of the epithelial-to-mesenchymal transition. J Pathol 2014, 232:319-329.

25. Korpal M, Lee ES, Hu G, Kang Y: The miR-200 family inhibits epithelialmesenchymal transition and cancer cell migration by direct targeting of E-cadherin transcriptional repressors ZEB1 and ZEB2. J Biol Chem 2008, 283:14910-14914.

26. Burk U, Schubert J, Wellner U, Schmalhofer O, Vincan E, Spaderna S, Brabletz $\mathrm{T}$ : A reciprocal repression between ZEB1 and members of the miR-200 family promotes EMT and invasion in cancer cells. EMBO Rep 2008, 9:582-589.

27. Bracken CP, Gregory PA, Kolesnikoff N, Bert AG, Wang J, Shannon MF, Goodall GJ: A double-negative feedback loop between ZEB1-SIP1 and the microRNA-200 family regulates epithelial-mesenchymal transition. Cancer Res 2008, 68:7846-7854.

28. Bracken CP, Li X, Wright JA, Lawrence DM, Pillman KA, Salmanidis M, Anderson MA, Dredge BK, Gregory PA, Tsykin A, et al.: Genome-wide identification of miR-200 targets reveals a regulatory network controlling cell invasion. EMBO J 2014, 33:2040-2056.

29. Brabletz S, Brabletz T: The ZEB/miR-200 feedback loop--a motor of cellular plasticity in development and cancer? EMBO Rep 2010, 11:670-677.

30. Tiwari N, Gheldof A, Tatari M, Christofori G: EMT as the ultimate survival mechanism of cancer cells. Semin Cancer Biol2012, 22:194-207.

31. Brabletz T, Hlubek F, Spaderna S, Schmalhofer O, Hiendlmeyer E, Jung A, Kirchner T: Invasion and metastasis in colorectal cancer: epithelialmesenchymal transition, mesenchymal-epithelial transition, stem cells and beta-catenin. Cells Tissues Organs 2005, 179:56-65.

32. Christofori G: New signals from the invasive front. Nature 2006, 441:444-450.

33. Puisieux A, Brabletz $T$, Caramel $\mathrm{J}$ : Oncogenic roles of EMT-inducing transcription factors. Nat Cell Biol 2014, 16:488-494.

34. Ye X, Weinberg RA: Epithelial-Mesenchymal Plasticity: A Central Regulator of Cancer Progression. Trends Cell Biol 2015, 25:675-686.

35. Yu M, Bardia A, Wittner BS, Stott SL, Smas ME, Ting DT, Isakoff SJ, Ciciliano JC, Wells MN, Shah AM, et al.: Circulating breast tumor cells exhibit dynamic changes in epithelial and mesenchymal composition. Science 2013, 339:580-584.

oo This report shows for the first time that mesenchymal gene expression is highly enriched in CTCs of breast cancer patients and correlates with poor clinical outcome, while only rare primary tumor cells simultaneously express epithelial and mesenchymal markers.

36. Kallergi G, Papadaki MA, Politaki E, Mavroudis D, Georgoulias V, Agelaki S: Epithelial to mesenchymal transition markers expressed in circulating tumour cells of early and metastatic breast cancer patients. Breast Cancer Res 2011, 13:R59.

37. Rhim AD, Mirek ET, Aiello NM, Maitra A, Bailey JM, McAllister F, Reichert M, Beatty GL, Rustgi AK, Vonderheide $\mathrm{RH}$, et al.: EMT and dissemination precede pancreatic tumor formation. Cell 2012, 148:349-361.

38. Prat A, Parker JS, Karginova O, Fan C, Livasy C, Herschkowitz JI, He X, Perou $\mathrm{CM}$ : Phenotypic and molecular characterization of the claudin-low intrinsic subtype of breast cancer. Breast Cancer Res 2010, 12:R68. 
39. Morel AP, Lievre M, Thomas C, Hinkal G, Ansieau S, Puisieux A: Generation of breast cancer stem cells through epithelial-mesenchymal transition. PLoS One 2008, 3:e2888.

40. Mani SA, Guo W, Liao MJ, Eaton EN, Ayyanan A, Zhou AY, Brooks M, Reinhard F, Zhang CC, Shipitsin M, et al.: The epithelial-mesenchymal transition generates cells with properties of stem cells. Cell 2008, 133:704-715.

41. Tsai JH, Donaher JL, Murphy DA, Chau S, Yang J: Spatiotemporal regulation of epithelial-mesenchymal transition is essential for squamous cell carcinoma metastasis. Cancer Cell 2012, 22:725-736.

42. Stankic M, Pavlovic S, Chin Y, Brogi E, Padua D, Norton L, Massague J, Benezra R: TGF-beta-Id1 signaling opposes Twist1 and promotes metastatic colonization via a mesenchymal-to-epithelial transition. Cell Rep 2013, 5:1228-1242.

43. Tran HD, Luitel K, Kim M, Zhang K, Longmore GD, Tran DD: Transient SNAIL1 expression is necessary for metastatic competence in breast cancer. Cancer Res 2014, 74:6330-6340.

44. Brabletz T, Jung A, Reu S, Porzner M, Hlubek F, Kunz-Schughart LA, Knuechel $\mathrm{R}$, Kirchner $\mathrm{T}$ : Variable beta-catenin expression in colorectal cancers indicates tumor progression driven by the tumor environment. Proc Natl Acad Sci U S A 2001, 98:10356-10361.

45. Brabletz T: To differentiate or not--routes towards metastasis. Nat Rev Cancer 2012, 12:425-436.

46. Jolly MK, Tripathi SC, Jia D, Mooney SM, Celiktas M, Hanash SM, Mani SA, Pienta KJ, Ben-Jacob E, Levine $\mathrm{H}$ : Stability of the hybrid epithelial/mesenchymal phenotype. Oncotarget 2016.

47. Harney AS, Arwert EN, Entenberg D, Wang Y, Guo P, Qian BZ, Oktay MH, Pollard JW, Jones JG, Condeelis JS: Real-Time Imaging Reveals Local, Transient Vascular Permeability, and Tumor Cell Intravasation Stimulated by TIE2hi Macrophage-Derived VEGFA. Cancer Discov 2015, 5:932-943.

o Using real-time microscopy analysis this study shows that tumor cell intravasation requires the local loss of vascular junctions elicited by the expression of VEGF-A from Tie2-expressing macrophages.

48. Entenberg D, Kedrin D, Wyckoff J, Sahai E, Condeelis J, Segall JE: Imaging tumor cell movement in vivo. Curr Protoc Cell Biol 2013, Chapter 19:Unit19 17.

49. Kang Y, Pantel K: Tumor cell dissemination: emerging biological insights from animal models and cancer patients. Cancer Cell 2013, 23:573-581.

50. Aceto N, Bardia A, Miyamoto DT, Donaldson MC, Wittner BS, Spencer JA, Yu M, Pely A, Engstrom A, Zhu $H$, et al.: Circulating tumor cell clusters are oligoclonal precursors of breast cancer metastasis. Cell 2014, 158:11101122.

oo In an elegant experimental approach, this study demonstrates that single cell CTCs are rare but more frequent than clusters of CTCs circulating in the bloodstream of cancer patients. Notably, CTC clusters are more efficient than single CTCs in forming experimental metastasis.

51. Sarioglu AF, Aceto N, Kojic N, Donaldson MC, Zeinali M, Hamza B, Engstrom A, Zhu H, Sundaresan TK, Miyamoto DT, et al.: A microfluidic device for label-free, physical capture of circulating tumor cell clusters. Nat Methods 2015, 12:685-691. 
o This manuscript reports the design and use of a microfluidic device for capturing CTCs independent of any marker expression from the blood of patients with a variety of cancer types.

52. Cheung KJ, Padmanaban V, Silvestri V, Schipper K, Cohen JD, Fairchild AN, Gorin MA, Verdone JE, Pienta KJ, Bader JS, et al.: Polyclonal breast cancer metastases arise from collective dissemination of keratin 14expressing tumor cell clusters. Proc Natl Acad Sci U S A 2016, 113:E854863.

oo Together with ref. 50 this study demonstrates that CTC clusters are polyclonal of origin and seed plolyclonal metastases in distant organs. The results suggest that CTC clusters have directly originated from the primary tumor possibly by collecive cell migration and have not formed by aggegration of single CTCs in the blood stream.

53. Au SH, Storey BD, Moore JC, Tang Q, Chen YL, Javaid S, Sarioglu AF, Sullivan $\mathrm{R}$, Madden MW, O'Keefe $\mathrm{R}$, et al.: Clusters of circulating tumor cells traverse capillary-sized vessels. Proc Natl Acad Sci U S A 2016.

54. Jolly MK, Boareto M, Huang B, Jia D, Lu M, Ben-Jacob E, Onuchic JN, Levine H: Implications of the Hybrid Epithelial/Mesenchymal Phenotype in Metastasis. Front Oncol 2015, 5:155.

55. Grosse-Wilde A, Fouquier d'Herouel A, Mclntosh E, Ertaylan G, Skupin A, Kuestner RE, del Sol A, Walters KA, Huang S: Stemness of the hybrid Epithelial/Mesenchymal State in Breast Cancer and Its Association with Poor Survival. PLoS One 2015, 10:e0126522.

56. Beerling E, Seinstra D, de Wit E, Kester L, van der Velden D, Maynard C, Schafer R, van Diest P, Voest E, van Oudenaarden A, et al.: Plasticity between Epithelial and Mesenchymal States Unlinks EMT from Metastasis-Enhancing Stem Cell Capacity. Cell Rep 2016, 14:2281-2288.

oo This study uses intravital imaging to demonstrate the existence of spontaneous EMT in breast tumors. Notably, the cells that have undergone an EMT need to revert to an epithelial differentiaton status to intiate metastatic outgrowth at the distant site.

57. Maddipati R, Stanger BZ: Pancreatic Cancer Metastases Harbor Evidence of Polyclonality. Cancer Discov 2015, 5:1086-1097.

58. Wicki A, Christofori G: The potential role of podoplanin in tumour invasion. $\mathrm{Br}$ $J$ Cancer 2007, 96:1-5.

59. Graves ML, Cipollone JA, Austin P, Bell EM, Nielsen JS, Gilks CB, McNagny KM, Roskelley $C D$ : The cell surface mucin podocalyxin regulates collective breast tumor budding. Breast Cancer Res 2016, 18:11.

60. Cheung KJ, Gabrielson E, Werb Z, Ewald AJ: Collective invasion in breast cancer requires a conserved basal epithelial program. Cell 2013, 155:1639-1651.

oo This report provides experimental evidence that collective invasion may underlie the metastatic dissemination of breast cancer cells, initiated and lead by cancer cells with a basal invasive gene expression program. 
61. Cheung KJ, Ewald AJ: A collective route to metastasis: Seeding by tumor cell clusters. Science 2016, 352:167-169.

62. Koelzer VH, Zlobec I, Lugli A: Tumor budding in colorectal cancer--ready for diagnostic practice? Hum Pathol 2016, 47:4-19.

63. Trimboli AJ, Fukino K, de Bruin A, Wei G, Shen L, Tanner SM, Creasap N, Rosol TJ, Robinson ML, Eng C, et al:: Direct evidence for epithelialmesenchymal transitions in breast cancer. Cancer Res 2008, 68:937-945.

64. Fischer KR, Durrans A, Lee S, Sheng J, Li F, Wong ST, Choi H, El Rayes T, Ryu $\mathrm{S}$, Troeger $\mathrm{J}$, et al.: Epithelial-to-mesenchymal transition is not required for lung metastasis but contributes to chemoresistance. Nature 2015, 527:472-476.

65. Zheng X, Carstens JL, Kim J, Scheible M, Kaye J, Sugimoto H, Wu CC, LeBleu VS, Kalluri R: Epithelial-to-mesenchymal transition is dispensable for metastasis but induces chemoresistance in pancreatic cancer. Nature 2015, 527:525-530.

oo Refs. 64 and 65 employ genetic lineage tracing and manipulation of the expression of EMT TFs, respectively, to demonstrate that EMT is not required for metastatic outgrowth. Yet, they report that cells that have undergone an EMT are resistant against chemotherapy and support metastasis formation upon chemotherapy.

66. Maheswaran S, Haber DA: Cell fate: Transition loses its invasive edge. Nature 2015, 527:452-453.

67. Zhao Z, Zhu X, Cui K, Mancuso J, Federley R, Fischer K, Teng G, Mittal V, Gao $D$, Zhao $H$, et al: In vivo visualization and characterization of epithelialmesenchymal transition in breast tumors. Cancer Res 2016, 76:20942104.

- Intravital microscopy of lineage tracing in transplanted breast tumors reveals the occurence of an EMT in primary tumors, which can be repressed by pharmacological inhibition of the HGF receptor c-Met.

\section{Figure Legend}

Schematic representation of the multiple stages of metastatic dissemination of cancer cells from the primary tumor into the blood circulation, dissemination through the vascular system, extravasation and formation of micrometastasis in a distant organ and, finally, outgrowth as macroscopic metastasis. As discussed in this review, the functional contribution of an EMT to the initial primary tumor cell invasion and intravasation and extravasation is not fully understood. While single circulating tumor cells (CTCs) are mainly exhibiting mesenchymal characteristics, CTC clusters that may have formed by collective migration and invasion may consist of cells with epithelial and mesenchymal marker expression. Disseminated tumor cells (DTCs) are found very early during tumorigenesis in distant organs and predominantly in the bone marrow. Moreover, EMT cells arriving at the metastatic side need to undergo a 
MET to outgrow as macrometastasis. On the other hand, EMT cells are more drug resistant and selected for by chemotherapy. 
Figure 1 Diepenbruck and Christofori

Primary tumor Primary tumor

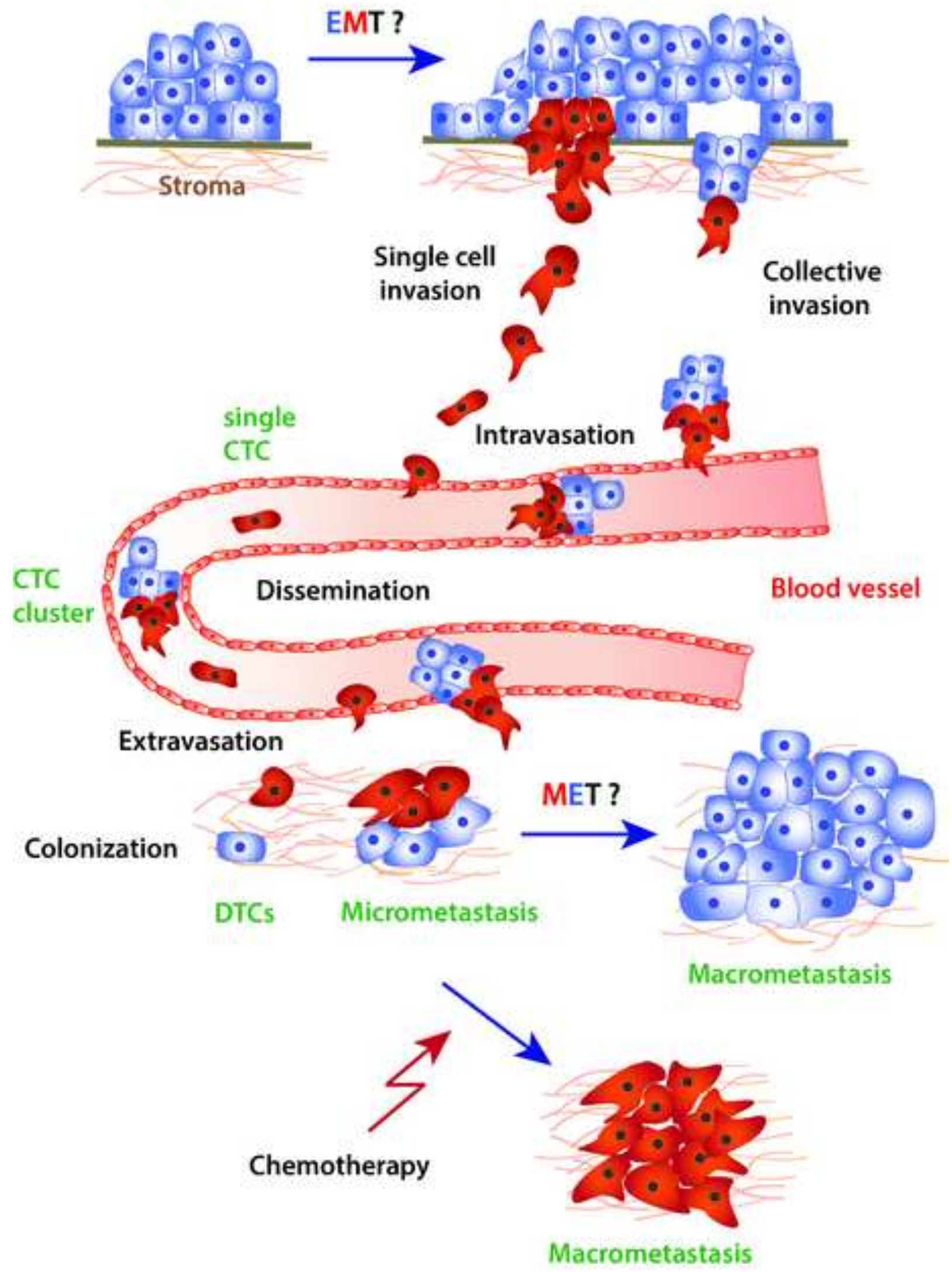

